\title{
Clinical Utility of Fedratinib in Myelofibrosis
}

\author{
Julian A Waksal \\ Douglas Tremblay (D) \\ John Mascarenhas
}

Department of Hematology and Oncology, Tisch Cancer Institute, Icahn School of Medicine at Mount Sinai, New York, NY, USA
Correspondence: John Mascarenhas Email John.Mascarenhas@mssm.edu

\begin{abstract}
Myelofibrosis (MF) is a clonal hematologic malignancy characterized by bone marrow fibrosis, extramedullary hematopoiesis, splenomegaly, and constitutional symptoms with a propensity towards leukemic transformation. Constitutive activation of the JAK/STAT pathway is a well-described pathogenic feature of MF. Allogeneic stem cell transplant is the only curative therapy, but due to high morbidity and mortality this option is not available for most patients. There are two approved targeted therapy options for MF, ruxolitinib and fedratinib. In this review, we discuss the clinical utility of fedratinib in the myelofibrosis treatment paradigm. Fedratinib has shown impressive pre-clinical and clinical efficacy in patients with untreated MF as well as in those with ruxolitinib intolerance and those with relapsed/refractory MF. Here, we review the pre-clinical and clinical trials that led to the approval of fedratinib, and the ongoing late-phase trials. We highlight several areas regarding the clinical utility of fedratinib that remain unanswered. We discuss the limitations of fedratinib and address areas that are understudied and require further clinical evaluation and research. The approval of fedratinib has provided a significant expansion to the very limited treatment armamentarium available to patients with MF.
\end{abstract}

Keywords: myelofibrosis, fedratinib, targeted therapy, JAK inhibitor

\section{Introduction}

Myelofibrosis (MF) is a myeloproliferative neoplasm (MPN) characterized by clonal proliferation of myeloid stem cells, bone marrow fibrosis, cytopenias, splenomegaly secondary to extramedullary hematopoiesis, and increased pro-inflammatory cytokine production with associated constitutional symptoms. ${ }^{1} \mathrm{MF}$ can arise de novo, called primary MF (PMF), or secondary to an antecedent MPN such as essential thrombocytosis (ET) or polycythemia vera (PV), termed post-ET MF or post-PV MF, respectively. ${ }^{2}$ Patients with $\mathrm{MF}$ have a propensity towards leukemic transformation, termed MPN blast phase (MPN-BP). ${ }^{3,4}$ A key pathogenic feature of MF is the constitutive activation of the JAK/STAT pathway, which is a result of a variety of well-described driver mutations including $J A K 2, C A L R$, or MPL. A mutation in one of these three genes is present in over $90 \%$ of patients with $\mathrm{MF}^{5}$ While the pathogenesis of MF is incompletely understood, constitutive activation of the JAK/STAT pathway is pivotal, leading to downstream signaling that stimulates cellular proliferation, impairs apoptosis, and promotes cytokine-independent and erythropoietin-independent colony formation. ${ }^{6,7}$ While allogeneic stem cell transplant (aSCT) is the only potentially curative therapeutic option for MF, treatment-related morbidity and mortality precludes this therapy in the majority of cases.

Given the central role of JAK-STAT overactivation in MF pathobiology, therapeutic targeting of this pathway has become the mainstay of pharmacologic 
treatment. Ruxolitinib (Jakafi, Incyte) is a selective JAK1 and JAK2 inhibitor approved by the Food and Drug Administration (FDA) in 2011 for treatment of patients with Dynamic International Prognostic Scoring System (DIPSS) intermediate or high-risk MF. Approval was based on the results of the COMFORT-I (NCT00952289). and COMFORT-II (NCT00934544) trials, which demonstrate activity in terms of spleen volume reduction (SVR) rate and improvement in constitutional symptom burden. ${ }^{8,9}$ Unfortunately, many patients may be intolerant to ruxolitinib, or become refractory. The median time to ruxolitinib discontinuation in long-term follow-up of patients enrolled in COMFORT-I and -II trials was 3 years. ${ }^{10}$ Discontinuation of ruxolitinib is a poor prognostic event, with a median survival of 11-15 months as demonstrated in multiple retrospective series. $^{11-13}$

Fedratinib (Inrebic, BMS) is a JAK2/FLT3 inhibitor that was FDA-approved in August 2019 based on the results of the JAKARTA and JAKARTA-2 studies. ${ }^{14,15}$ Fedratinib had a clinical development delay due to a prolonged full clinical hold from 2013 to 2017 related to several cases concerning drug-related Wernicke encephalopathy (WE). ${ }^{16}$ Further review led to a lift of this hold and ultimate approval of fedratinib for the treatment of MF patients either in the front-line or after ruxolitinib discontinuation, fulfilling an urgent need.

In this review, we detail the clinical utility of fedratinib in the treatment of patients with MF. We first describe the benefits and limitations of ruxolitinib, the standard firstline JAK inhibitor. We then delve into the pre-clinical and clinical trial data that led to the approval of fedratinib and how this agent fits into the current treatment paradigm. We then describe the limitations with this JAK inhibitor and consider the future role of fedratinib for the treatment of $\mathrm{MF}$.

\section{Ruxolitinib}

Ruxolitinib is a JAK1/JAK2 inhibitor that was approved based on results of the COMFORT-I and II trials. COMFORT-I was a double-blind, placebo-controlled trial which randomized 309 patients with International Prognostic Scoring System (IPSS) intermediate-2 or high-risk MF to twice daily ruxolitinib or placebo. The primary endpoint was SVR of $\geq 35 \%\left(\mathrm{SVR}_{35 \%}\right)$ at 24 weeks, assessed by magnetic resonance imaging. In the ruxolitinib arm, $42 \%$ of patients achieved the primary endpoint compared to $<1 \%$ in the placebo arm.
Additionally, in those who had a response, the reduction in spleen volume was durable, with $67 \%$ of responding patients maintaining a response at 48 weeks. Regarding secondary endpoints, $46 \%$ of those in the treatment arm had a decrease in MF symptom assessment form (MF$\mathrm{SAF})$ total symptom score of $>50 \%\left(\mathrm{TSS}_{50 \%}\right)$, compared to $5 \%$ of those in the placebo arm at the 24-week endpoint. Reduction in spleen volumes and improvements in TSS were seen in both wild-type (WT) and mutated $J A K 2$. Additionally, overall survival (OS) was shown to be improved in the ruxolitinib group at median follow-up period of 51 weeks, with a hazard ratio of $0.5(95 \%$ CI 0.25-0.98). Ruxolitinib was well tolerated, with anemia and thrombocytopenia being the most common grade 3 or 4 adverse event (AE), as well as the most common indications for dose reduction or discontinuation. ${ }^{9}$

The COMFORT-II trial randomized 219 patients with IPSS intermediate-2 or high-risk primary, post-PV, or postET MF in a 2:1 ratio to receive ruxolitinib or best available therapy (BAT). Similar to the COMFORT-I trial the primary outcome was $\mathrm{SVR}_{35 \%}$, but in COMFORT-II this was measured at 48 weeks, with 24-week spleen measurement as a secondary outcome. Dose adjustments were made for patients with platelet counts $<200 \times 10^{9} / \mathrm{L}$ to $15 \mathrm{mg}$ twice a day in place of the $20 \mathrm{mg}$ twice a day dose for those with platelet counts $>200 \times 10^{9} / \mathrm{L}$. The most common BAT given to patients was hydroxyurea in $47 \%$ of patients and glucocorticoids in $16 \%$ of enrolled patients. $\mathrm{SVR}_{35 \%}$ was observed in $28 \%$ of subjects in the ruxolitinib arm versus $0 \%$ in the BAT arm. There was $\mathrm{TSS}_{50 \%}$ observed in $46 \%$ of subjects receiving ruxolitinib versus $5 \%$ in the BAT arm. A long-term follow-up pooled analysis of patients enrolled in COMFORT-I and II trials support the OS benefit in patients treated with ruxolitinib with median OS of 5.3 years compared to 2.4 years in the control group. ${ }^{10}$ Adverse event profile was similar to that seen in the COMFORT-I trial, with anemia and thrombocytopenia being the most common AEs. ${ }^{8}$ Further supporting the efficacy demonstrated in the pivotal trials in the United States, the JUMP trial enrolled 2233 patients in countries without access to ruxolitinib outside of clinical trials and included patients with thrombocytopenia and without splenomegaly. Though this was a non-randomized trial, it supports the results of the COMFORT-I/II trials in this patient population, leading to significant improvements in splenomegaly, as well as symptoms in patients with and without splenomegaly at baseline. The adverse event profile suggests that ruxolitinib has a similar safety 
profile in patients with thrombocytopenia $\left(<200 \times 10^{9} / \mathrm{L}\right.$, $\left.>50 \times 10^{9} / \mathrm{L}\right)$ compared to those with normal platelet counts. ${ }^{17}$

Because crossover was allowed from the placebo to the treatment arm in the COMFORT-I and II trials, the rankpreserving structural failure time (RPSFT) was utilized to calculate the OS hazard ratio. While there may be a true survival benefit, as suggested by the study, the RPSFT is a theoretical model that utilizes statistical assumptions. ${ }^{18}$ The COMFORT-I and II trials were not statistically powered to detect a difference in OS benefit due to the short follow-up time and small number of events at the time of analysis. ${ }^{19}$ Additionally, data from long-term follow-up studies of the COMFORT trials are difficult to interpret due to lack of randomization, or controls. The OS benefit was further investigated in a Cochrane systematic review evaluating the efficacy of ruxolitinib, which concludes that there is insufficient evidence to draw conclusions on survival benefit compared to placebo or BAT. ${ }^{20}$ Accordingly, we must interpret the OS benefit noted in the COMFORT-I and II trials with caution, and this remains a topic of debate.

Further limitation of ruxolitinib includes its utility in patients with disease-related cytopenias. Given that thrombopoietin and erythropoietin receptors are JAK/STATdependent, ruxolitinib often leads to on-target anemia and thrombocytopenia. In the COMFORT-I trial, grade 3/ 4 anemia and thrombocytopenia were observed in $43 \%$ and $17 \%$ of ruxolitinib-treated patients, respectively. ${ }^{9,21}$ Therefore, patients with a platelet count of $<50 \times 10^{9} / \mathrm{L}$ are ineligible for ruxolitinib, and dose reduction is recommended for patients with platelet counts $<200 \times 10^{9} / \mathrm{L} .{ }^{22}$ In addition, due to its potential to exacerbate disease-related anemia, ruxolitinib should be used with caution in patients with anemia. Additionally, long-term follow-up studies have demonstrated that ruxolitinib may decrease progression of fibrosis in the marrow at 60 months follow-up, and may improve the odds of reduction of bone marrow fibrosis, although the clinical significance of this effect remains uncertain. ${ }^{23}$ Ruxolitinib does not reduce leukemic progression, neither in long-term follow-up of the COMFORT studies nor in pre-clinical modeling. ${ }^{24}$ Moreover, nearly all patients will eventually develop resistance or progress to MPN-BP, or develop intolerance (most frequently secondary to dose-limiting cytopenias) leading to discontinuation within a median of three years of initiation therapy. ${ }^{12}$ Importantly, abrupt discontinuation of ruxolitinib has been associated with a withdrawal syndrome thought to be secondary to an acute cytokine rebound leading to a septic-shock-like picture, and in some cases respiratory distress. ${ }^{25}$ Discontinuation of ruxolitinib has been associated with rapid recurrence of symptoms, overall poor outcomes, and an OS of 11-15 months. ${ }^{11-13}$ Until the approval of fedratinib in 2019, there was no evidence that supported second-line pharmacologic agents for patients with refractory spleen or symptoms or those who were intolerant to ruxolitinib.

\section{Pre-clinical Development of Fedratinib}

In 2005 , the gain-of-function $J A K 2 V 617 F$ mutation was first identified and noted to be highly prevalent in patients with MPNs. ${ }^{26}$ There was almost immediate interest in the JAK/STAT pathway as a therapeutic target in MF, and JAK2 inhibitors were developed and quickly entered clinical testing. ${ }^{27}$ TG10148, which would later be named fedratinib, was among the early compounds identified as a JAK2 inhibitor. This agent was initially designed as a targeted JAK2 inhibitor by optimizing a compound identified in a kinase-inhibitor library utilizing rational structure-based techniques. Fedratinib was selected for clinical development due to its low $\mathrm{IC}_{50}$ of $3 \mathrm{nM}$ to JAK2, indicative of its highly potent characteristics. ${ }^{28}$ Fedratinib was also shown to have off-target inhibition of both mutated and wild-type FLT3, a kinase that phosphorylates and activates multiple pathways associated with anti-apoptosis and cellular proliferation at relevant dosing concentrations. $^{28,29}$ Targeted FLT3 inhibitors have demonstrated significant survival benefit in the treatment of FLT3-mutated acute myeloid leukemia, ${ }^{30,31}$ although the biologic impact of this FLT3 inhibition in MF, where activating mutations are not typically seen, is underexplored.

In addition, fedratinib has shown inhibitor activity against BRD4, a member of the bromodomain and extraterminal domain (BET) protein family. BET proteins have been shown to play a fundamental role in cellular proliferation and division as well as pro-inflammatory signaling. ${ }^{32,33}$ Inhibition of JAK/STAT pathway and BET inhibition has been shown to decrease cytokine production and reverse bone marrow fibrosis in mouse models. ${ }^{34}$ Therefore, it is possible that the dual-kinase activity against JAK2 and BRD4 contribute to the clinical efficacy profile of fedratinib. 
In pre-clinical models, fedratinib was evaluated in a $J A K 2 V 617 F$ retrovirus-induced MPN murine system, which demonstrated dose-dependent reduction in erythrocytosis and splenomegaly. Additionally, histopathological analysis of splenic and hepatic tissue from the murine model showed markedly reduced extramedullary hematopoiesis in mice treated with fedratinib compared to those treated with placebo. ${ }^{28}$ In contrast to the decrease in allele burden seen in the $J A K 2 V 617 F$ retrovirus-induced murine model, no decrease in allele burden was noted in a mutant allele knock-in mouse model. ${ }^{35}$ A subsequent murine model was conducted to evaluate the effect of fedratinib in varying disease phenotypes including post-PV MF and post-PV MF. The post-PV model was developed from $J A K 2 V 617 F$ knock-in mice, while the post-ET MF model was developed by continuous MPL activation. Fedratinib sharply decreased spleen weight in all models. No decrease in allele burden was observed in the $J A K 2 V 617 F$ knock-in model. Interestingly, bone marrow fibrosis and osteosclerosis was significantly suppressed in the post-PV MF model, but not in the post-ET MF model. ${ }^{36}$ In summary, although the effect on allele burden and bone marrow fibrosis was equivocal depending on mechanism of mutant allele introduction into murine models, fedratinib showed significant pre-clinical efficacy across MPN phenotypes.

\section{Phase I Study of Fedratinib}

In a multicenter phase 1 study of fedratinib in patients with intermediate or high-risk primary or post-PV/ET MF, 59 patients were enrolled, with 28 patients in the dose-escalation phase and 31 in the dose-confirmation phase. The maximally tolerated dose (MTD) was determined to be $680 \mathrm{mg} /$ day based on reversible hyperamylasemia that occurred in two patients at $800 \mathrm{mg} /$ day. Grade 3 nonhematologic AEs were almost exclusively reported in the MTD cohort and included nausea, diarrhea, and vomiting, which were low grade, self-limited, and dose-dependent. Grade 3-4 hematologic events that occurred were anemia $(35 \%)$, thrombocytopenia $(24 \%)$, or neutropenia $(10 \%)$. Fedratinib also demonstrated promising evidence of clinical activity. After 12 cycles, more than half of patients reported improvement in constitutional symptoms including early satiety, fatigue night sweats, cough, and pruritus, which were evaluated on a 10-point scale at each visit. Spleen responses, defined as $\geq 50 \%$ reduction in spleen size by palpation, were observed in $47 \%$ of patients. Of the 23 patients harboring $\geq 20 \% J A K 2 V 617 F$ allele burden, the median allele burden at baseline for this group was $60 \%$. After 12 cycles of treatment, the median allele burden in this group was $32 \%{ }^{37}$

In this phase 1 study, with fedratinib administered to patients at doses of 30-800 $\mathrm{mg} / \mathrm{day}$, the mean steady-state maximum plasma concentration $\left(\mathrm{C}_{\max }\right)$ increased 54-fold and area under the concentration-time curve $\left(\mathrm{AUC}_{0-\mathrm{t}}\right)$ values increased 88 -fold over a 27 -fold increase in dose. $^{37}$ The half-life of fedratinib is 41 hours, and steady-state plasma concentrations were achieved by day 15. Fedratinib is metabolized by CYP3A4, CYP2C19, and FMO3. The terminal phase half-life of fedratinib at steadystate was 16 to 34 hours across all doses tested. This is consistent with linear drug elimination. In two healthy volunteer phase 1 studies, the bioavailability of fedratinib was not affected by food intake, and the tolerability was improved when taken after consumption of a high-fat meal. $^{38}$

Given the promising safety and efficacy profile, as well as the potentially disease-modifying effect as implicated by the decreased allele burden, fedratinib moved forward to late-phase clinical trials in patients with MF.

\section{Phase 2 Dose-Finding Study of Fedratinib}

In 2015, Pardanani and colleagues published the results of a phase 2 randomized dose-finding study evaluating 31 patients with intermediate- 2 or high-risk MF who received either fedratinib $300 \mathrm{mg}, 400 \mathrm{mg}$, or $500 \mathrm{mg}$ daily. The primary endpoint was $\mathrm{SVR}_{35 \%}$ at 12 weeks measured by computed tomography or magnetic resonance imaging. At 12 weeks, the rates of $\mathrm{SVR}_{35 \%}$ were $30 \%, 50 \%$, and $64 \%$ in the $300 \mathrm{mg}, 400 \mathrm{mg}$, and $500 \mathrm{mg}$ cohort, respectively. By 24 weeks, improvements in the MF-SAF TSS were observed across all doses. The proportion of patients with a $\mathrm{TSS}_{50 \%}$ at 24 weeks was $33 \%, 60 \%$, and $38 \%$ in the 300 , 400 , and $500 \mathrm{mg}$ dose groups, respectively. Contrary to the phase 1 trial, patients with $J A K V 617 F$ were not shown to have a statistically significant reduction in allele burden, with median change in allele burden of $-4.57 \%,-0.05 \%$, and $-8.16 \%$ in the 300,400 , and $500 \mathrm{mg}$ groups, although the trial was not powered to detect an effect in this endpoint. At 48 weeks, $68 \%$ of patients remained on fedratinib while $16 \%$ discontinued due to AEs, and the rest of patients discontinued due to withdrawal of consent, withholding of drug for $\geq 8$ weeks, investigator discretion, or for personal reasons. Six patients (19\%) who were 
Table I Rates of Grade III/IV Adverse Events Across Phase 3 Trials in JAK-Inhibitor-Naïve Patients

\begin{tabular}{|c|c|c|c|c|}
\hline Trial & Discontinuation Rate & Diarrhea & Anemia & Thrombocytopenia \\
\hline JAKARTA $^{14 *}$ & $14 \%$ & $5 \%$ & $43 \%$ & $17 \%$ \\
\hline COMFORT I** & $11 \%$ & $1.90 \%$ & $45.2 \%$ & $12.90 \%$ \\
\hline COMFORT II*** & NR & $1 \%$ & $42 \%$ & $8 \%$ \\
\hline
\end{tabular}

Notes: ${ }^{\#}$ Discontinuation rate at 24 weeks due to adverse events. *Fedratinib $400 \mathrm{mg}$ dose cohort, $n=96$. **Comfort I: NCT00952289. Ruxolitinib variable doses depending on plt count, $n=155$. ***Comfort II: NCT00934544. Ruxolitinib variable doses depending on plt count, $n=\mid 46$.

transfusion-independent at baseline developed red blood cell (RBC) transfusion dependence. The most common indications for dose reductions during the trial were anemia and elevated lipase level. Of note, one patient in the $300 \mathrm{mg}$ group developed mildly elevated transaminases and was dose-reduced to $200 \mathrm{mg}$ but then subsequently developed sub-massive hepatic necrosis with reversible liver failure upon discontinuation of therapy. One case of WE was observed and confirmed by MRI in the $500 \mathrm{mg}$ group in a 70-year-old woman with a history of chronic diarrhea, significant weight loss, and cachexia predating study enrollment and an ischemic stroke while on trial the week preceding the diagnosis of WE. ${ }^{39}$ The decision to move forward with the $400 \mathrm{mg}$ and $500 \mathrm{mg}$ daily dose regimens in later-phase trials was based on the improved efficacy with significant improvements in spleen size and symptom burden in the higher-dose cohorts with a similar side effect profile and need for dose reductions between all dosing groups.

\section{JAKARTA Study in Ruxolitinib- Naïve Patients}

The JAKARTA trial, a phase 3 trial, evaluated 289 patients with DIPSS intermediate-2 or high-risk primary MF, post$\mathrm{PV}$, and post-ET MF randomized to receive fedratinib at $400 \mathrm{mg} /$ day, $500 \mathrm{mg} /$ day, or placebo in a 1:1:1 fashion. At baseline, $63 \%$ of patients enrolled had primary MF, $10 \%$ had post-ET MF, and $27 \%$ had post-PV MF. Regarding risk status, $52 \%$ of patients had DIPSS intermediate- 2 risk MF and 48\% had high-risk MF. Eighty-two of 289 (28\%) of patients were $J A K 2 \mathrm{WT}$, while the rest (72\%) had $J A K 2 V 617 F$ mutant MF. The $500 \mathrm{mg}$ arm had a higher proportion of $J A K 2 V 617 F$ mutations than the $400 \mathrm{mg}$ or placebo group (78\% vs $65 \%$ vs $61 \%$ ). Otherwise, baseline characteristics were relatively equally distributed across study arms. The primary endpoint was $\mathrm{SVR}_{35 \%}$ at week 24 with a secondary endpoint of $\mathrm{TSS}_{50 \%}$. The primary endpoint was met in $36 \%$ and $40 \%$ in the $400 \mathrm{mg}$ and $500 \mathrm{mg}$ cohort, respectively, while only $1 \%$ of patients achieved a spleen response in the placebo arm. Additionally, the proportion of patients achieving $\mathrm{TSS}_{50 \%}$ was $36 \%$ and $34 \%$ in patients treated with fedratinib at $400 \mathrm{mg}$ or $500 \mathrm{mg}$ dose, respectively, compared with $7 \%$ in those in the placebo arm. There is a comparable response rate in terms of $\mathrm{SVR}_{35 \%}$ and $\mathrm{TSS}_{50 \%}$ across phase 3 trials evaluating ruxolitinib and fedratinib at the approved dose (400 mg) (Table 1). Of note, in the subgroup analysis, fedratinib demonstrated significant efficacy irrespective of $J A K 2$ mutational status. Among fedratinibtreated patients, $77 \%$ of patients enrolled completed 24 weeks of treatment, with $82 \%$ of patients in the $400 \mathrm{mg}$ daily dose arm reaching this milestone and $71 \%$ of patients in the $500 \mathrm{mg}$ daily dose arm. No meaningful changes were observed in the $J A K V 617 F$ allele burden during treatment across all study arms. At 24 weeks, $73 \%$ of patients in the placebo arm crossed over to fedratinib, as allowed per protocol due to progressive disease. The most common AEs leading to discontinuation were thrombocytopenia, diarrhea, and vomiting. Of note, GI toxicity led to a dose interruption or dose adjustment in $20 \%$ of patients enrolled. Importantly, 4 cases of WE were reported exclusively in the $500 \mathrm{mg} /$ day cohort, with 3 cases confirmed by imaging. Otherwise, the toxicity profile was similar to that observed in the phase 2 trial above, with anemia being the most common hematologic AE. ${ }^{14}$ The toxicity profile and rate of discontinuation at 24 weeks across phase 3 trials evaluating ruxolitinib and fedratinib are shown in Table 2.

Table 2 Response Rates Across Phase 3 Trials in JAK-InhibitorNaïve Patients

\begin{tabular}{|c|c|c|}
\hline Trial & $\mathbf{S V R}_{\mathbf{3 5}} \%$ & TSS $_{50 \%}$ \\
\hline JAKARTA $^{14 *}$ & $36 \%$ & $36 \%$ \\
\hline COMFORT I** & $42 \%$ & $46 \%$ \\
\hline COMFORT II*** & $32 \%$ & NR \\
\hline
\end{tabular}

Notes: *Fedratinib $400 \mathrm{mg}$ dose cohort, $n=96$. **Comfort I: NCT00952289. Ruxolitinib variable doses depending on plt count, $n=155$. *** Comfort II: NCT00934544. Ruxolitinib variable doses depending on plt count, $n=\mid 46$. Abbreviations: SVR $35 \%$, splenic volume reduction of $\geq 35 \%$; TSS50\%, MPN-total symptom score improvement by $\geq 50 \%$. 


\section{JAKARTA-2 Study in Ruxolitinib Intolerance or Resistance}

Given the efficacy of fedratinib in the front-line setting, the logical next step was to evaluate this therapy in patients previously treated with ruxolitinib. The JAKARTA-2 trial was a single-arm open-label, nonrandomized phase 2 multicenter trial that evaluated the use of fedratinib in patients with intermediate or high-risk primary MF or secondary MF who are either resistant to or intolerant of ruxolitinib. The criteria for resistance were lack of response, stable disease, evidence of disease progression, or loss of response to $\geq 14$ days of ruxolitinib. The criteria for intolerance to ruxolitinib were defined by discontinuation due to unacceptable toxicity after any duration on ruxolitinib. It is important to note that this trial included intermediate-1 risk patients with constitutional symptoms, while the larger phase 3 JAKARTA study did not. In the 83 assessable patients, $\mathrm{SVR}_{35 \%}$ was observed in $55 \%$ of subjects. In 90 patients evaluable, symptom response evaluated by $\mathrm{TSS}_{50 \%}$ was achieved in $26 \%$ of subjects. Toxicity profile was consistent with previously discussed studies. The drug was well tolerated, with $19 \%$ of patients discontinuing treatment due to AEs; however, the full clinical hold due to cases of WE that were observed in the JAKARTA trial led to premature termination of this trial and the inability to follow-up for long-term survival analysis. ${ }^{8}$

Because the definition for ruxolitinib refractoriness was poorly defined in this study, a post-hoc analysis of the JAKARTA-2 trial evaluated 79 patients that met a stringent definition of relapse/refractory $(\mathrm{R} / \mathrm{R})$ to ruxolitinib. Here, relapse was defined as ruxolitinib treatment for $\geq 3$ months with spleen regrowth, defined as SVR $<10 \%$, or $<30 \%$ decrease in baseline spleen size following an initial response. Refractory was defined as $\geq 3$ months of treatment with ruxolitinib with SVR $<10 \%$ or $<30 \%$ decrease in spleen size from baseline. Intolerance was defined as development of red blood cell transfusion requirement or grade 3 or higher hematologic $\mathrm{AE}$ on $\geq 28$ days on ruxolitinib. In this analysis, 79 patients met the criteria for $\mathrm{R} / \mathrm{R}$ or intolerance. Twenty-four (30\%) patients achieved a $\mathrm{SVR}_{35 \%}$, reaffirming the meaningful potential benefit in this patient population. Symptom response rate in the posthoc analysis was $27 \%$, consistent with data presented in the original trial. ${ }^{40}$

\section{Wernicke Encephalopathy and Full Clinical Hold}

In 2013, 8 of 670 patients across ongoing late phase trials evaluating fedratinib were reported to have experienced neurologic complications of concern for possible WE. Of eight patients with neurologic symptoms suggestive of WE, seven were female, six had MF, one had PV, and one had metastatic head and neck cancer. With concern for a potentially life-threatening drug-related toxicity, the FDA placed a clinical hold on fedratinib on November 15, 2013. In terms of proposed mechanisms leading to WE, fedratinib has been shown to cross the blood-brain barrier in animal models and has been shown to inhibit human thiamine transporter (hTHTR2) in vitro, leading to theoretically decreased gut absorption. ${ }^{41,42}$ However, given the low rate of confirmed WE seen across all fedratinib trials, it is more likely that this neurologic toxicity is related to underlying thiamine or nutritional deficiencies in the study population. It has been shown that patients with MPN are at higher risk for developing WE than the general population, unrelated to use of fedratinib. ${ }^{43}$ Further, it has been shown in a prospective analysis that subnormal thiamine levels are a rare occurrence in MPN patients, and fedratinib does not lead to decreased thiamine levels. ${ }^{44}$ Of the eight possible cases, further review of data from 3 patients revealed that thiamine levels and MRI results were not supportive of WE and neurologic complications were determined to be due to non-drug related etiology. One patient was determined to have definite WE, 2 with likely WE, and 2 with an inconclusive diagnosis of WE. ${ }^{45}$ With 5 cases in 670 patients $(0.7 \%)$, the overall prevalence of WE in this study population is less than or equal to the estimated rate of $0.4-2.8 \%$ in the general population. ${ }^{46}$ During the FDA clinical hold, Sanofi, which had purchased fedratinib from TargeGen in 2010, discontinued the drug candidate from their pipeline and halted all plans for further development. In October 2017, Impact Biomedicines, founded by the former research chief and co-inventor of fedratinib at TargeGen, purchased the full rights for global development and commercialization of the drug candidate from Sanofi (Figure 1).

It was not until January 2018 that the FDA completed its review of the cases of possible WE, and it was determined that all patients who suffered this neurologic effect had cachexia or other nutritional risk factors for development of a thiamine deficiency. The complication was determined to be preventable with thiamine supplementation in those at high risk, and the clinical hold was lifted. 


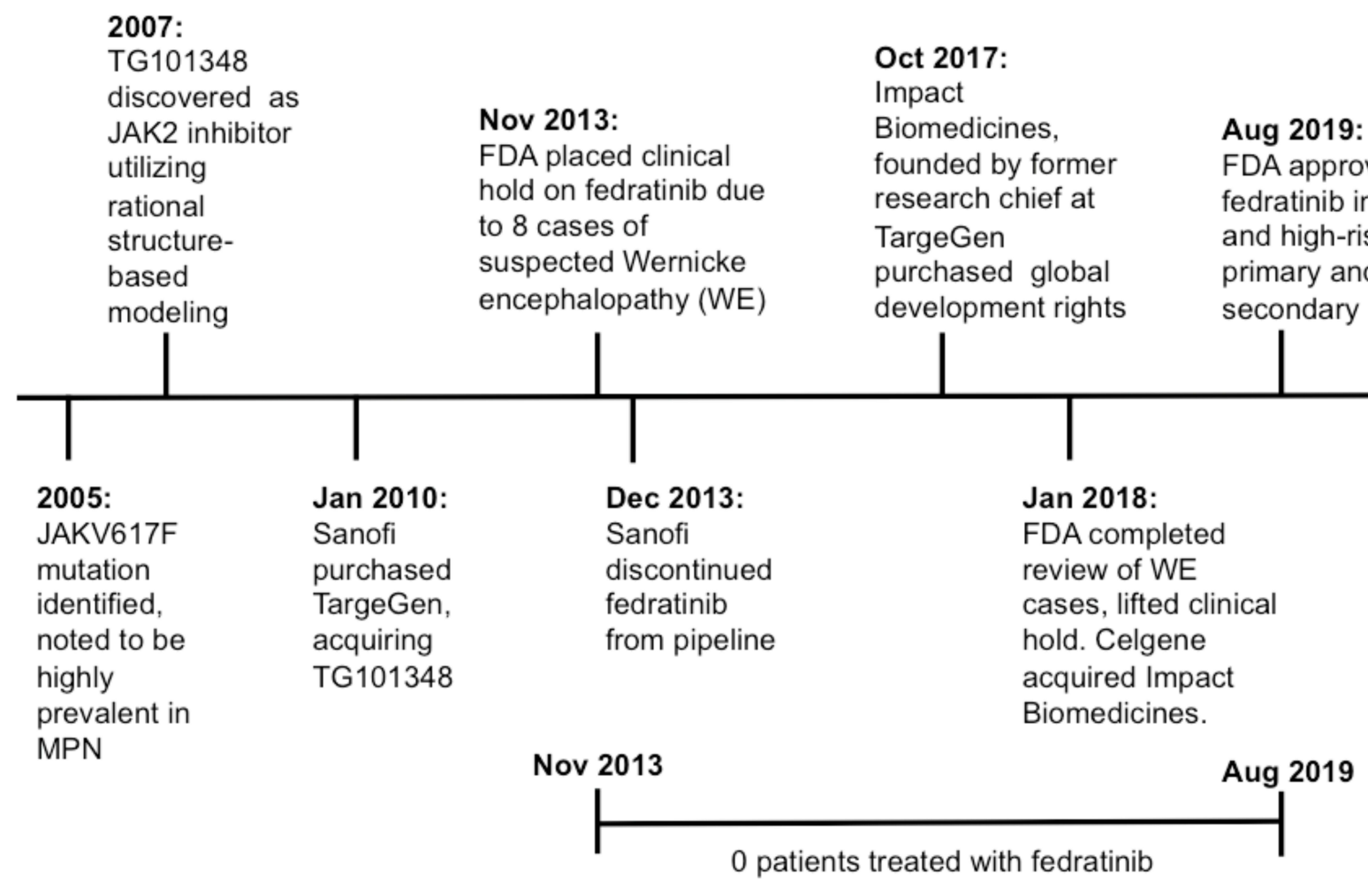

Figure I Timeline of fedratinib development and clinical hold.

Nevertheless, fedratinib has a black box warning for risk of WE. To prevent this complication, providers must check thiamine levels prior to starting therapy, and intermittently throughout therapy course. Fedratinib should be held if patients are thiamine-deficient until thiamine levels have been replenished. ${ }^{47}$

After a thorough re-analysis of cases of WE, fedratinib was FDA-approved in August 2019 for treatment of DIPSS intermediate-2 or high-risk primary or secondary MF. Importantly, this approval allows for fedratinib to be employed in patients without ruxolitinib exposure as well as in patients who are intolerant, refractory, or relapsed after ruxolitinib therapy. ${ }^{47}$ Interestingly, FDA approval was based on the results of the JAKARTA and JAKARTA-2 trials (Table 3) that had been conducted while in development at Sanofi and terminated due to concern for WE. The approval at this time was in the hands of Celgene and was achieved without enrolling a single additional patient.

\section{Current Clinical Application of Fedratinib}

While the approval of fedratinib has produced an urgently needed commercially available treatment option for MF patients who are intolerant, relapsed, or refractory to ruxolitinib, it has not necessarily significantly changed standard of care in the front-line setting. Clinicians have the option to prescribe either fedratinib or ruxolitinib for patients with DIPSS intermediate-2 or high-risk MF (Figure 2). There are no published data evaluating the efficacy of fedratinib compared with that of ruxolitinib at this time. Unlike ruxolitinib, fedratinib is not approved for intermediate-1 risk MF, although this is not necessarily a limitation in clinical practice. As a front-line agent, fedratinib, as recently suggested by long-term follow-up data, has progression-free survival (PFS) benefit. For those treated with fedratinib as first-line therapy, median PFS was 23.2 months versus 17.5 months in the placebo arm. Median OS was not reached in either trial arm. ${ }^{48}$ Longer follow-up studies are needed to determine OS benefit of fedratinib in the upfront setting.

Regarding therapy choice of a front-line agent in those with DIPSS intermediate-2 or high-risk MF, National Cancer Center Network (NCCN) guidelines recommend use of either ruxolitinib as category $2 \mathrm{~A}$, or fedratinib as category $2 \mathrm{~B}$. Because of the black box warning for WE that remains, the GI toxicity profile, and the lack of comparative study of ruxolitinib versus fedratinib as 


\begin{tabular}{|c|c|c|c|c|}
\hline 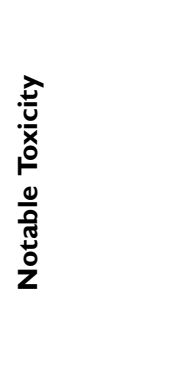 & 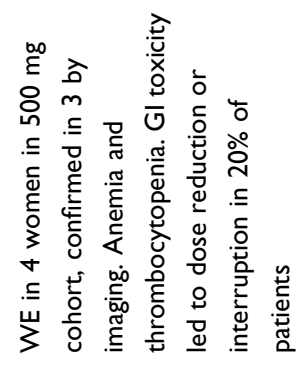 & 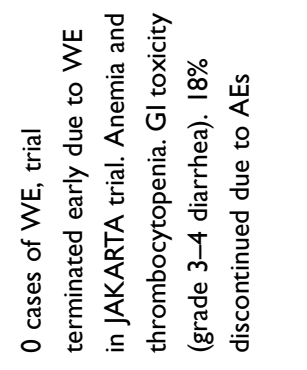 & 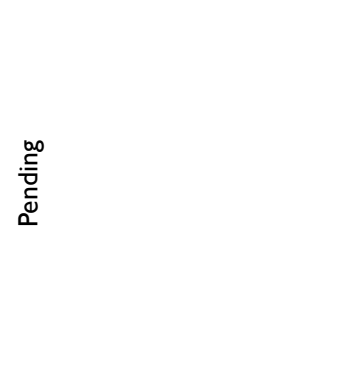 & 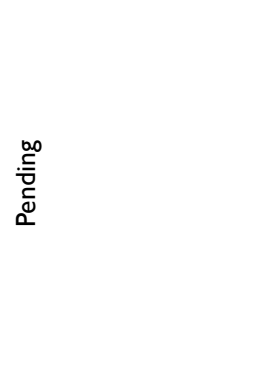 \\
\hline 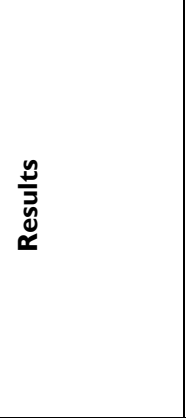 & 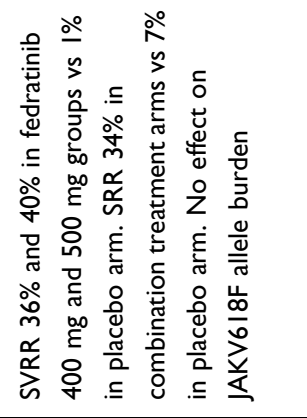 & 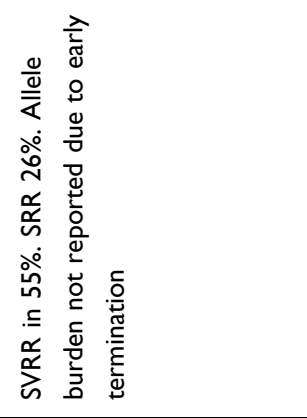 & 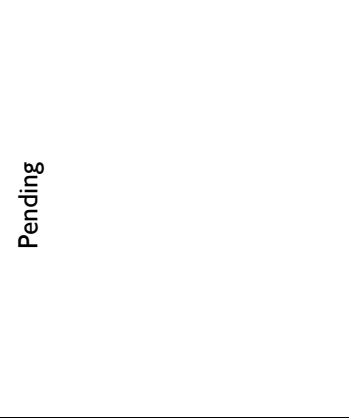 & 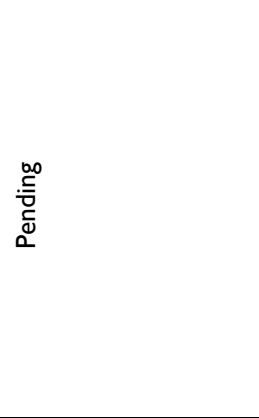 \\
\hline 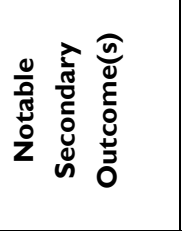 & 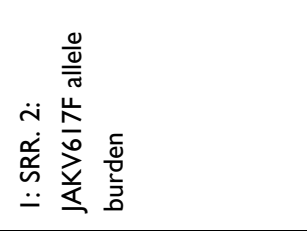 & 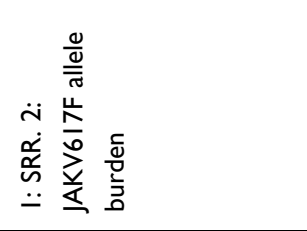 & 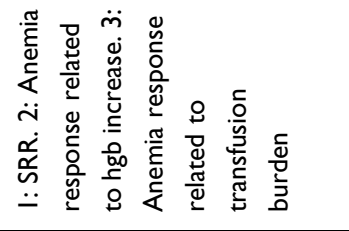 & $\begin{array}{l}\mathscr{0} \\
\dot{\sim} \\
\tilde{\alpha} \\
\tilde{x} \\
\ddot{=}\end{array}$ \\
\hline 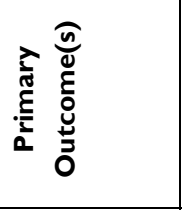 & 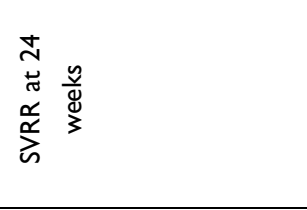 & 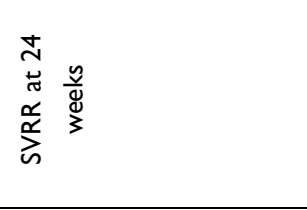 & 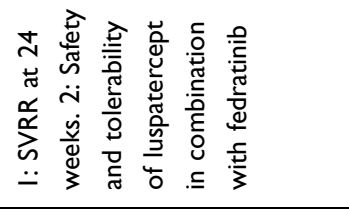 & 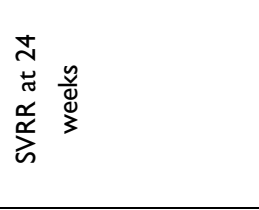 \\
\hline 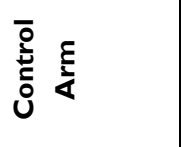 & 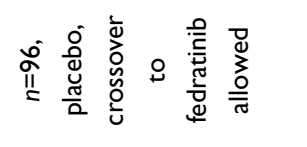 & $\begin{array}{l}\stackrel{0}{\tilde{0}} \\
\text { ż }\end{array}$ & $\begin{array}{l}\stackrel{0}{0} \\
\stackrel{0}{0}\end{array}$ & 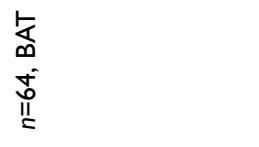 \\
\hline 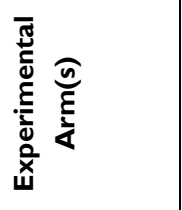 & 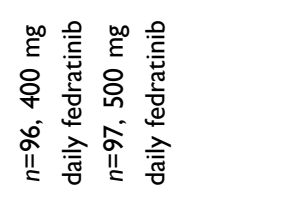 & 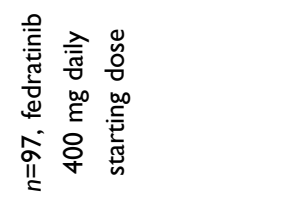 & 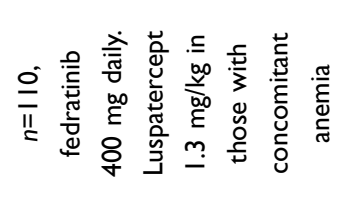 & 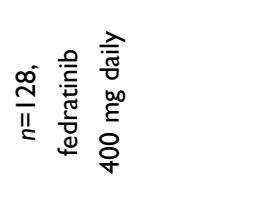 \\
\hline 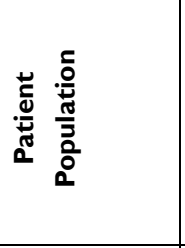 & 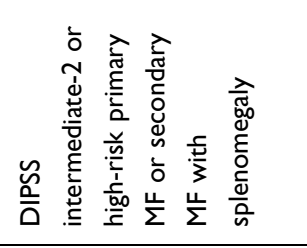 & 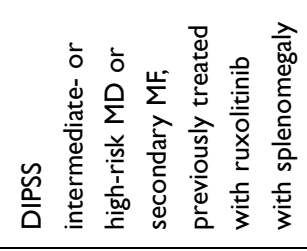 & 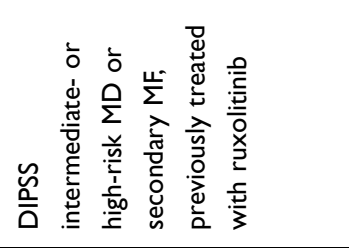 & 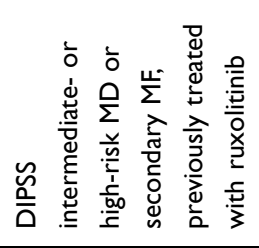 \\
\hline 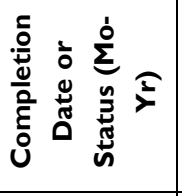 & $\begin{array}{l}\frac{\nabla}{\dot{s}} \\
\stackrel{5}{\underline{s}}\end{array}$ & $\frac{\frac{\pi}{\frac{1}{2}}}{\frac{1}{\alpha}}$ & 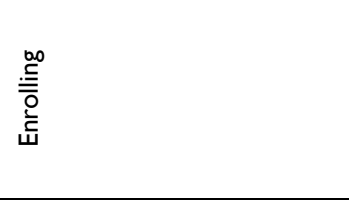 & 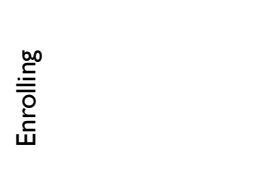 \\
\hline 晃 & 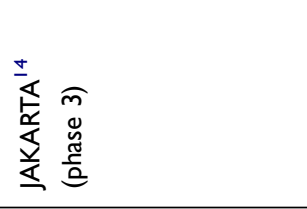 & 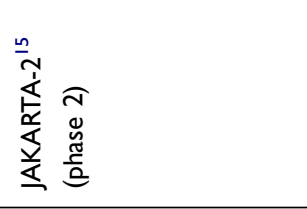 & 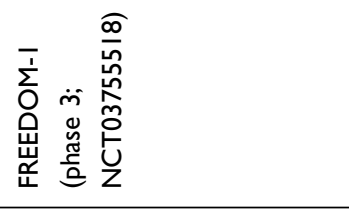 & 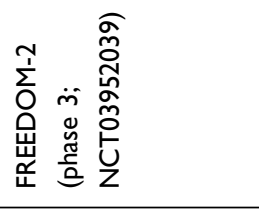 \\
\hline
\end{tabular}




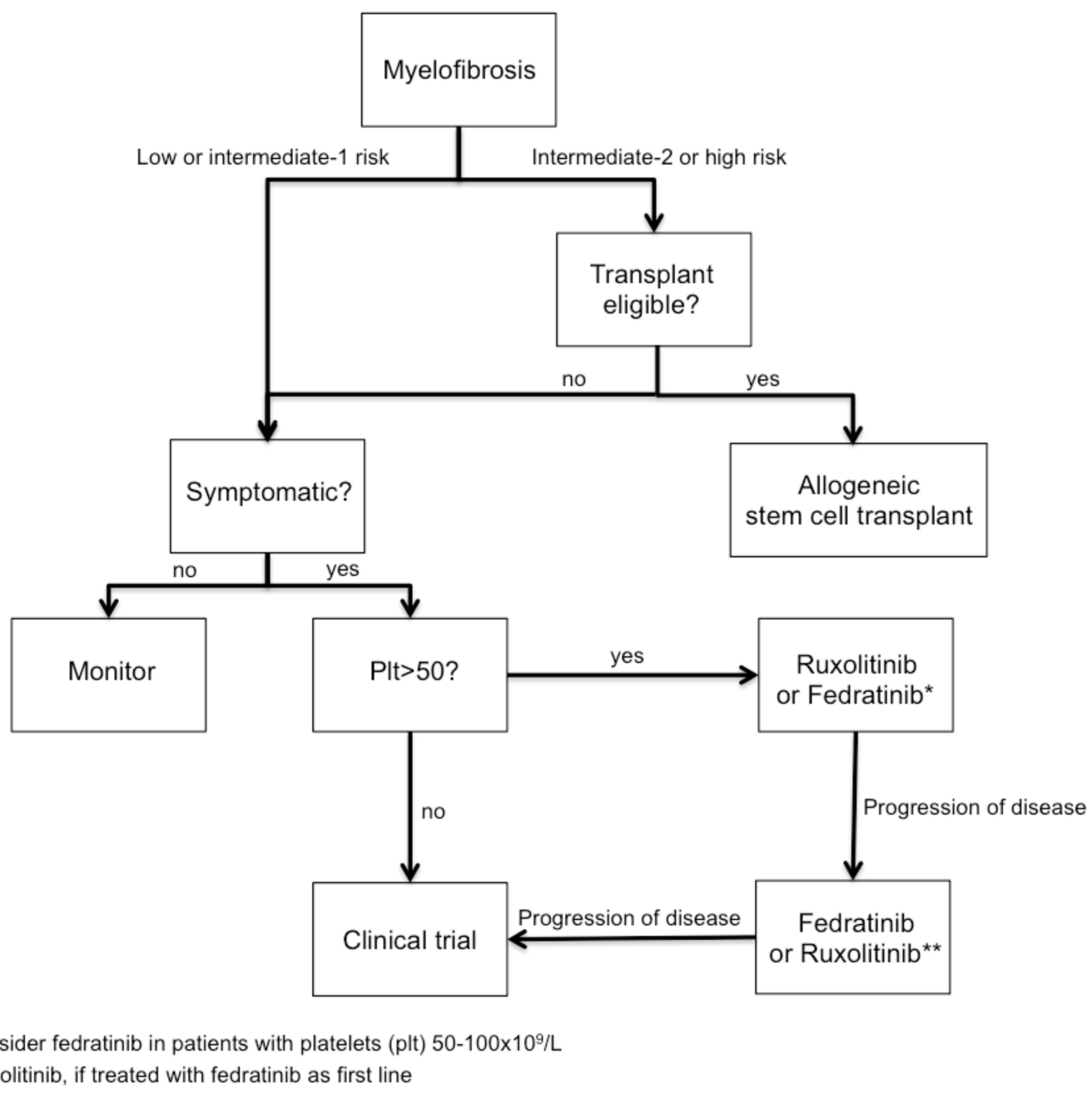

Figure 2 Myelofibrosis treatment algorithm.

a front-line therapy, ruxolitinib remains the preferred treatment of choice in this setting until more real-world data evaluating clinical benefit of fedratinib are available. Patients and clinicians may prefer the metabolic profile of fedratinib, given that ruxolitinib has been shown to cause considerable weight gain, while fedratinib has not been shown to have this effect. ${ }^{49,50}$ As discussed above, longterm data are suggestive of significant clinical benefit, particularly a prolonged PFS, of fedratinib, but these data are still not robust enough to indicate which JAK inhibitor is more effective when used as front-line agent. As the OS benefits of fedratinib are further studied, frontline therapy choice may shift from ruxolitinib to fedratinib.

Long-term outcomes of the JAKARTA-2 study were also recently reported. The median OS was not reached, and the 18 month survival rate was $67 \%$, which compares favorably with historically poor outcomes of patients after ruxolitinib discontinuation, previously observed to have median OS of 11 months. In addition, median PFS was 13.3 months. Importantly, survival analysis was censored at the time of full clinical hold. ${ }^{11,51}$ Further outcome studies are ongoing in patients receiving fedratinib after discontinuation of ruxolitinib (NCT03755518, NCT03952039). Importantly, the ability to accurately evaluate effect on overall survival in long-term follow-up studies was impacted by the clinical hold that was placed on fedratinib, which truncated survival follow-up. The ongoing FREEDOM trials, discussed below, will help further evaluate survival outcomes of those treated with fedratinib in subjects previously treated with ruxolitinib (Table 3).

When choosing between JAK inhibitor options, it is important to note that while the recommended dose of ruxolitinib is adjusted for platelet counts less than $200 \times 10^{9} / \mathrm{L}$, and further dose reductions are recommended 
for platelet counts $<100 \times 10^{9} / \mathrm{L}$, this is not the case for fedratinib. In a post-hoc analysis of the JAKARTA and JAKARTA-2 trials, the safety and efficacy of $400 \mathrm{mg}$ fedratinib was evaluated in patients with platelets $>50 \times 10^{9} / \mathrm{L}$ and $<100 \times 10^{9} / \mathrm{L}$. In those with baseline platelet count $<100 \times 10^{9} / \mathrm{L}$, the $\mathrm{SVR}_{35 \%}$ rate was significantly higher in the fedratinib group than that in the placebo group (36\% versus $0 \%$ ). Additionally, the proportion of patients achieving $\mathrm{TSS}_{50 \%}$ was significantly higher in the treatment group than the placebo arm, 35\% and $0 \%$, respectively. Across all patients in both JAKARTA and JAKARTA-2 trials, thrombocytopenia led to drug discontinuation in only 1 patient with baseline platelet count $<100 \times 10^{9} / \mathrm{L} .{ }^{52}$ These data suggest that the recommended dose of $400 \mathrm{mg} /$ day dose in patients with thrombocytopenia $50-100 \times 10^{9} / \mathrm{L}$ has similar safety and efficacy profiles in those with platelet counts $>100 \times 10^{9} / \mathrm{L}$. If platelet counts drop below $<50 \times 10^{9} / \mathrm{L}$, discontinuation of fedratinib is recommended until platelets rise above this threshold, and resumption at $100 \mathrm{mg}$ below prior daily dose is standard protocol. In patients who develop new transfusion dependence, it is recommended to consider a dose reduction by $100 \mathrm{mg}$ below prior daily dose. ${ }^{47}$ Importantly, neither fedratinib nor ruxolitinib are recommended for patients with baseline platelet counts $<50 \times 10^{9} / \mathrm{L}$, which remains an area of significant unmet clinical need.

\section{Limitations}

Fedratinib has undoubtedly created a long-needed, effective second-line option for MF and has changed the treatment algorithm for MF (Figure 2); however, the updated treatment armamentarium still leaves several important unmet needs. While fedratinib has produced clinically meaningful reduction in spleen size and symptom burden, like ruxolitinib, fedratinib has not been shown to be curative. In a pre-clinical MPN murine model, lethally irradiated mice received a bone marrow transplant from donor mice treated with fedratinib and developed an MPN phenotype similar to that seen in the primary donor mice, suggesting that fedratinib does not eradicate MPN-propagating stem cells. ${ }^{53}$ Further, it remains unclear how fedratinib impacts disease course in patients. Animal models have demonstrated that mice treated with fedratinib have decreased $J A K 2 V 617 F$ allele burden, but this remains a debated and unclear topic with conflicting data presented in humans, as discussed above. ${ }^{37,39,53}$ Additionally, while trial data are very promising for SVR and symptom burden improvement, we do not have real- world efficacy data, which are often significantly less impressive than clinical trial data.

Further limitations of fedratinib include its toxicity profile. More than $20 \%$ of patients in the JAKARTA trial developed GI toxicity including diarrhea, nausea, or vomiting. ${ }^{14}$ While this did not lead to frequent dose adjustment or discontinuation in this late-phase trial, this may impact compliance or preference for another treatment option in routine clinical practice. Additionally, treatment of disease-related cytopenias in patients receiving fedratinib is difficult given the frequent hematologic complications of JAK2 inhibition, anemia, and thrombocytopenia. Like ruxolitinib, fedratinib does not improve diseaserelated cytopenias. It is possible that a combination of fedratinib with agents that address disease-related anemia (eg, danazol, lenalidomide, or luspatercept) might be a future therapeutic option, although this has not been formally explored to date. However, there are ongoing clinical trials that will elucidate the best way to manage MF-related anemia in patients receiving fedratinib (NCT03755518).

\section{Future Clinical Trials of Fedratinib in MF}

The ongoing FREEDOM-1 trial is a single-arm, open-label safety and efficacy trial in patients with DIPSS intermediate or high-risk primary or secondary MF who have been previously treated with ruxolitinib. This trial will expand our understanding of safety and efficacy in this patient population. Additionally, this study will include a substudy in subjects with anemia, combining fedratinib plus luspatercept to evaluate the safety and tolerability of the combination (NCT03755518). The ongoing FREEDOM-2 is a phase $3 \mathrm{~b}$, randomized study evaluating fedratinib versus BAT in subjects with intermediate or high-risk primary or secondary MF who have been previously treated with ruxolitinib for at least 3 months with relapsed or refractory disease, or more than 28 days with intolerance to ruxolitinib (NCT03952039). These trials include patients with DIPSS intermediate-1 risk MF, while the pivotal JAKARTA trial did not include this risk cohort. These trials will help further clarify the safety and efficacy of fedratinib in relapsed/refractory/intolerant disease. Furthermore, the FREEDOM-1 trial will help us better understand the safety of managing disease-related anemia with luspatercept when treating patients with fedratinib. 
Further studies are needed to guide clinicians in their treatment choices for patients with MF. As previously mentioned, head-to-head comparison of fedratinib and ruxolitinib in the upfront setting is not available and is unlikely to ever be performed. Recent data suggest that patients who have developed intolerance or relapse on ruxolitinib may benefit from a washout period, followed by re-introduction of ruxolitinib. ${ }^{54,55}$ Reintroduction of ruxolitinib in those who have relapsed or developed intolerance versus treatment with fedratinib has also not been studied. Additionally, there are no data to suggest a safe protocol for transitioning from ruxolitinib to fedratinib. Our current practice is to taper ruxolitinib over 5 days and start $400 \mathrm{mg}$ fedratinib on day 3 of the taper, therefore overlapping therapy for 3 days. This is not guideline- or data-driven.

With the development and introduction of novel therapies, clinical questions regarding optimal treatment paradigms will continue to arise. Several novel therapies in the pipeline are being evaluated as add-on therapies to ruxolitinib in patients with MF. Parsaclisib (NCT04551053), navitoclax (NCT04472598), KRT-232 (NCT04485260), and pelabresib (NCT02158858) are all in clinical-stage development and being evaluated in combination with ruxolitinib. Further studies will need to be conducted to evaluate how fedratinib performs in patients who have relapsed after these combination regimens. Additionally, it is unclear how the lack of JAK1 inhibition with fedratinib impacts the disease biology and clinical durability of response. Similarly, the impact of FLT3 inhibition of MF disease biology is poorly understood. Finally, predictors of response, including molecular and cytogenetic factors, remain unknown. Further studies, hopefully in the realworld setting, will be needed to advance our understanding of this important agent for the treatment of MF.

\section{Future of MF Therapy}

Beyond fedratinib and ruxolitinib, several other therapies are in late-stage development, both as monotherapies and in combination with ruxolitinib, as noted above. Other JAK inhibitors that have shown promise include momelotinib and pacritinib. Momelotinib is a JAK1/2 and ACVR1 inhibitor being studied in the MOMENTUM trial, which is particularly well suited for patients with disease-related anemia (NCT04173494). Pacritinib is primarily an equipotent JAK2/FLT3/IRAK1 inhibitor that is being studied in the phase 3 PACIFICA trial in patients with platelet count $<50 \times 10^{9} / \mathrm{L}$ (NCT03165734).
In addition to JAK inhibitors, several other novel targeted therapies have shown promising data and are being developed in the JAK inhibitor-naïve setting and in patients previously treated with ruxolitinib. Targeted therapies that have reached late-phase development include BCL2 (navitoclax), PI3K (parsaclisib), telomerase (imetelstat), BET (pelabresib), and MDM2 (KRT-232) inhibitors. ${ }^{56}$ With each approval, the landscape and treatment paradigms for MF will rapidly shift.

While the mainstay of treatment for many hematologic malignancies are combination regimens, there are currently no approved combination therapies approved for MF. Thus far in MF therapeutic development, combination therapies have been limited by the number of approved therapies that function in a single pathway. As discussed, there are several ongoing trials evaluating ruxolitinib in combination with novel therapies (NCT03222609, NCT03194542, NCT02158858). If these studies show promising data, combination therapies will likely become the standard of care for treatment of MPNs. Fedratinib will need to be further studied in combination with already-approved therapies such as luspatercept to manage disease-related anemia, as well as with novel targeted therapies to better control disease. As more drugs and combination regimens are developed and approved, we hope to study and understand more about predictors of response to each therapy target, which will further guide our treatment sequencing and determine the optimal treatment for patients with MF.

\section{Conclusions}

With the profound clinical benefit demonstrated in pivotal trials, fedratinib is an exciting development and therapeutic option for patients with intermediate- 2 or high-risk MF in both the upfront setting and in those previously treated with ruxolitinib. Information on the performance of fedratinib outside of the carefully controlled clinical trial setting is needed to confirm the value of this agent. Currently, this agent is the only commercially available targeted therapy for MF patients who have discontinued ruxolitinib. With the introduction of novel therapies, especially those with targets outside the JAK/STAT pathway, the role of fedratinib in the MF treatment paradigm is less certain. For now, we look forward to results of ongoing and future clinical trials, as well as long-term real-world data, that will further inform the use of fedratinib for the treatment of MF. 


\section{Disclosure}

Dr John Mascarenhas reports grants and/or consulting fees from Incyte, Roche, Geron, Constellation, PharmaEssentia, BMS, Celgene, Kartos, CTIBiopharma, Merck, Forbius, Premedior, Novartis, and Janssen, outside the submitted work. The authors report no other conflicts of interest in this work.

\section{References}

1. Tefferi A. Primary myelofibrosis: 2019 update on diagnosis, risk-stratification and management. Am J Hematol. 2018;93 (12):1551-1560. doi:10.1002/ajh.25230

2. O'Sullivan JM, Harrison CN. Myelofibrosis: clinicopathologic features, prognosis, and management. Clin Adv Hematol Oncol. 2018;16 (2):121-131

3. Tefferi A, Guglielmelli P, Larson DR, et al. Long-term survival and blast transformation in molecularly annotated essential thrombocythemia, polycythemia vera, and myelofibrosis. Blood. 2014;124(16):2507-13; quiz 2615. doi:10.1182/blood-2014-05579136

4. Vallapureddy RR, Mudireddy M, Penna D, et al. Leukemic transformation among 1306 patients with primary myelofibrosis: risk factors and development of a predictive model. Blood Cancer J. 2019;9 (2):12. doi:10.1038/s41408-019-0175-y

5. Tefferi A, Lasho TL, Finke CM, et al. CALR vs JAK2 vs MPL-mutated or triple-negative myelofibrosis: clinical, cytogenetic and molecular comparisons. Leukemia. 2014;28(7):1472-1477. doi:10.1038/leu.2014.3

6. da Costa Reis Monte-mór B, Plo I, da Cunha AF, et al. Constitutive JunB expression, associated with the JAK2 V617F mutation, stimulates proliferation of the erythroid lineage. Leukemia. 2009;23 (1):144-152. doi:10.1038/leu.2008.275

7. Walz C, Crowley BJ, Hudon HE, et al. Activated Jak2 with the $\mathrm{V} 617 \mathrm{~F}$ point mutation promotes $\mathrm{G} 1 / \mathrm{S}$ phase transition. J Biol Chem. 2006;281(26):18177-18183. doi:10.1074/jbc.M6000 64200

8. Harrison C, Kiladjian JJ, Al-Ali HK, et al. JAK inhibition with ruxolitinib versus best available therapy for myelofibrosis. $N$ Engl J Med. 2012;366(9):787-798. doi:10.1056/NEJMoa1110556

9. Verstovsek S, Mesa RA, Gotlib J, et al. A double-blind, placebo-controlled trial of ruxolitinib for myelofibrosis. $N$ Engl $J$ Med. 2012;366(9):799-807. doi:10.1056/NEJMoa1110557

10. Verstovsek S, Gotlib J, Mesa RA, et al. Long-term survival in patients treated with ruxolitinib for myelofibrosis: COMFORT-I and -II pooled analyses. J Hematol Oncol. 2017;10(1):156. doi:10.1186/ s13045-017-0527-7

11. Mascarenhas J, Mehra M, He J, Potluri R, Loefgren C. Patient characteristics and outcomes after ruxolitinib discontinuation in patients with myelofibrosis. J Med Econ. 2020;23(7):721-727. doi:10.1080/13696998.2020.1741381

12. Palandri F, Breccia M, Bonifacio M, et al. Life after ruxolitinib: reasons for discontinuation, impact of disease phase, and outcomes in 218 patients with myelofibrosis. Cancer. 2020;126(6):1243-1252. doi: $10.1002 /$ cncr.32664

13. Newberry KJ, Patel K, Masarova L, et al. Clonal evolution and outcomes in myelofibrosis after ruxolitinib discontinuation. Blood. 2017;130(9):1125-1131. doi:10.1182/blood-2017-05-783225

14. Pardanani A, Harrison C, Cortes JE, et al. Safety and efficacy of fedratinib in patients with primary or secondary myelofibrosis: a randomized clinical trial. JAMA oncol. 2015;1(5):643-651. doi:10.1001/jamaoncol.2015.1590
15. Harrison CN, Schaap N, Vannucchi AM, et al. Janus kinase-2 inhibitor fedratinib in patients with myelofibrosis previously treated with ruxolitinib (JAKARTA-2): a single-arm, open-label, non-randomised, phase 2, multicentre study. Lancet Haematol. 2017;4(7):e317-e324. doi:10.1016/S2352-3026(17)30088-1

16. Center for drug evaluation and research multi-discipline review, Application Number: 212327Orig1s000; 2019. Available from: https://www.accessdata.fda.gov/drugsatfda_docs/nda/2019/ 212327Orig1s000MultidisciplineR.pdf. Accessed August 16, 2021.

17. Al-Ali HK, Griesshammer M, le Coutre P, et al. Safety and efficacy of ruxolitinib in an open-label, multicenter, single-arm phase $3 \mathrm{~b}$ expanded-access study in patients with myelofibrosis: a snapshot of 1144 patients in the JUMP trial. Haematologica. 2016;101 (9):1065-1073. doi:10.3324/haematol.2016.143677

18. Francisco Cervantes AP. Does ruxolitinib prolong the survival of patients with myelofibrosis?. Blood. 2017; 129(7):832-837.. doi:10.1182/blood-2016-11-731604d

19. Cervantes F, Pereira A. Does ruxolitinib prolong the survival of patients with myelofibrosis? Blood. 2017;129(7):832-837. doi:10.1182/blood-2016-11-731604

20. Marti-Carvajal AJ, Anand V, Sola I. Janus kinase-1 and Janus kinase-2 inhibitors for treating myelofibrosis. Cochrane Database Syst Rev. 2015;(4):CD010298. doi:10.1002/14651858.CD010298.pub2

21. Levine RL, Pardanani A, Tefferi A, Gilliland DG. Role of JAK2 in the pathogenesis and therapy of myeloproliferative disorders. Nat Rev Cancer. 2007;7(9):673-683. doi:10.1038/nrc2210

22. Ruxolitinib [package insert]. Wilmington, DE: Incyte Corporation; 2011.

23. Kvasnicka HM, Thiele J, Bueso-Ramos CE, et al. Long-term effects of ruxolitinib versus best available therapy on bone marrow fibrosis in patients with myelofibrosis. J Hematol Oncol. 2018;11(1):42. doi:10.1186/s13045-018-0585-5

24. Mylonas E, Yoshida K, Frick M, et al. Single-cell analysis based dissection of clonality in myelofibrosis. Nat Commun. 2020;11(1):73. doi:10.1038/s41467-019-13892-x

25. Tefferi A, Pardanani A. Serious adverse events during ruxolitinib treatment discontinuation in patients with myelofibrosis. Mayo Clin Proc. 2011;86(12):1188-1191. doi:10.4065/mcp.2011.0518

26. Baxter EJ, Scott LM, Campbell PJ, et al. Acquired mutation of the tyrosine kinase JAK2 in human myeloproliferative disorders. Lancet. 2005;365(9464):1054-1061. doi:10.1016/S0140-6736(05) $71142-9$

27. Zaleskas VM, Krause DS, Lazarides K, et al. Molecular pathogenesis and therapy of polycythemia induced in mice by JAK2 V617F. PLoS One. 2006;1(1):e18. doi:10.1371/journal.pone.0000018

28. Wernig G, Kharas MG, Okabe R, et al. Efficacy of TG101348, a selective JAK2 inhibitor, in treatment of a murine model of JAK2V617F-induced polycythemia vera. Cancer Cell. 2008;13 (4):311-320. doi:10.1016/j.ccr.2008.02.009

29. Kiyoi H, Ohno R, Ueda R, Saito H, Naoe T. Mechanism of constitutive activation of FLT3 with internal tandem duplication in the juxtamembrane domain. Oncogene. 2002;21(16):2555-2563. doi:10.1038/sj.onc. 1205332

30. Stone RM, Mandrekar SJ, Sanford BL, et al. Midostaurin plus chemotherapy for acute myeloid leukemia with a FLT3 mutation. $N$ Engl J Med. 2017;377(5):454-464. doi:10.1056/NEJMoa1614359

31. Perl AE, Martinelli G, Cortes JE, et al. Gilteritinib or chemotherapy for relapsed or refractory. $N$ Engl J Med. 2019;381(18):1728-1740. doi:10.1056/NEJMoa1902688

32. Ciceri P, Muller S, O'Mahony A, et al. Dual kinase-bromodomain inhibitors for rationally designed polypharmacology. Nat Chem Biol. 2014;10(4):305-312. doi:10.1038/nchembio.1471

33. Jiang Q, Jamieson C. BET'ing on dual JAK/BET inhibition as a therapeutic strategy for myeloproliferative neoplasms. Cancer Cell. 2018;33(1):3-5. doi:10.1016/j.ccell.2017.12.007 
34. Kleppe M, Koche R, Zou L, et al. Dual targeting of oncogenic activation and inflammatory signaling increases therapeutic efficacy in myeloproliferative neoplasms. Cancer Cell. 2018;33(4):785-787. doi:10.1016/j.ccell.2018.03.024

35. Mullally A, Poveromo L, Schneider RK, Al-Shahrour F, Lane SW, Ebert BL. Distinct roles for long-term hematopoietic stem cells and erythroid precursor cells in a murine model of Jak2V617F-mediated polycythemia vera. Blood. 2012;120(1):166-172. doi:10.1182/blood2012-01-402396

36. Debeurme F, Lacout C, Moratal C, et al. JAK2 inhibition has different therapeutic effects according to myeloproliferative neoplasm development in mice. J Cell Mol Med. 2015;19(11):2564-2574. doi: $10.1111 / \mathrm{jcmm} .12608$

37. Pardanani A, Gotlib JR, Jamieson C, et al. Safety and efficacy of TG101348, a selective JAK2 inhibitor, in myelofibrosis. J Clin Oncol. 2011;29(7):789-796. doi:10.1200/JCO.2010.32.8021

38. Zhang M, Xu C, Ma L, et al. Effect of food on the bioavailability and tolerability of the JAK2-selective inhibitor fedratinib (SAR302503) results from two phase I studies in healthy volunteers. Clin Pharmacol Drug Dev. 2015;4(4):315-321. doi:10.1002/cpdd.161

39. Pardanani A, Tefferi A, Jamieson C, et al. A phase 2 randomized dose-ranging study of the JAK2-selective inhibitor fedratinib (SAR302503) in patients with myelofibrosis. Blood Cancer J. 2015;5(8):e335. doi:10.1038/bcj.2015.63

40. Harrison CN, Schaap N, Vannucchi AM, et al. Fedratinib in patients with myelofibrosis previously treated with ruxolitinib: an updated analysis of the JAKARTA2 study using stringent criteria for ruxolitinib failure. Am J Hematol. 2020;95(6):594-603. doi:10.1002/ajh.25777

41. Zhang $\mathrm{M}$, Xu CR, Shamiyeh $\mathrm{E}$, et al. A randomized, placebo-controlled study of the pharmacokinetics, pharmacodynamics, and tolerability of the oral JAK2 inhibitor fedratinib (SAR302503) in healthy volunteers. J Clin Pharmacol. 2014;54 (4):415-421. doi:10.1002/jcph.218

42. Zhang Q, Zhang Y, Diamond S, et al. The Janus kinase 2 inhibitor fedratinib inhibits thiamine uptake: a putative mechanism for the onset of wernicke's encephalopathy. Drug Metab Dispos. 2014;42 (10):1656-1662. doi:10.1124/dmd.114.058883

43. Wu J, Zhang L, Vaze A, Lin S, Juhaeri J. Risk of wernicke's encephalopathy and cardiac disorders in patients with myeloproliferative neoplasm. Cancer Epidemiol. 2015;39(2):242-249. doi:10.1016/j.canep.2015.01.014

44. Gangat N, Phelps A, Lasho TL, et al. A prospective evaluation of vitamin B1 (thiamine) level in myeloproliferative neoplasms: clinical correlations and impact of JAK2 inhibitor therapy. Blood Cancer J. 2019;9(2):11. doi:10.1038/s41408-018-0167-3

45. Claire N, Harrison RAM, Jamieson C, et al. Case series of potential wernicke's encephalopathy in patients treated with fedratinib. Blood. 2017. doi:10.1182/blood.V130.Suppl_1.4197.4197Sd
46. Ota Y, Capizzano AA, Moritani T, Naganawa S, Kurokawa R, Srinivasan A. Comprehensive review of wernicke encephalopathy: pathophysiology, clinical symptoms and imaging findings. Jpn $J$ Radiol. 2020;38(9):809-820. doi:10.1007/s11604-020-00989-3

47. Fedratinib [package insert]. Summit (NJ): Celgene Corporation; 2019.

48. Harrison CN, Schaap N, Vannucchi AM, et al. Fedratinib induces spleen responses and reduces symptom burden in patients with Myeloproliferative Neoplasm (MPN)-associated Myelofibrosis (MF) and low platelet counts, who were either ruxolitinib-naïve or were previously treated with ruxolitinib. Blood. 2019. doi:10.1182/blood2019-129288Sd

49. Sapre M, Tremblay D, Wilck E, et al. Metabolic effects of JAK1/2 inhibition in patients with myeloproliferative neoplasms. Sci Rep. 2019;9(1):16609. doi:10.1038/s41598-019-53056-x

50. D T, L C, SR B, S R, J M. The effect of fedratinib, a selective inhibitor of Janus Kinase 2 (JAK2), on weight and metabolic parameters in patients with intermediate (INT)- or high-risk Myelofibrosis (MF). Blood. 2020;136:16-17.

51. Harrison C, Kiladjian J, Verstovsek S, et al. Overall and progression-free survival in patients treated with fedratinib as first-line Myelofibrosis (MF) therapy and after prior Ruxolitinib (RUX): results from the jakarta and jakarta2 trials; 2021.

52. Harrison CN, Schaap N, Vannucchi AM, Kiliadjian J-J. Fedratinib induces spleen responses and reduces symptom burden in patients with Myeloproliferative Neoplasm (MPN)-associated Myelofibrosis (MF) and low platelet counts, who were either ruxolitinib-naïve or were previously treated with ruxolitinib. Blood. 2019. doi:10.1182/ blood-2019-129288

53. Mullally A, Lane SW, Ball B, et al. Physiological Jak2V617F expression causes a lethal myeloproliferative neoplasm with differential effects on hematopoietic stem and progenitor cells. Cancer Cell. 2010;17(6):584-596. doi:10.1016/j.ccr.2010.05.015

54. Gerds A, Su D, Martynova A, et al. Ruxolitinib rechallenge can improve constitutional symptoms and splenomegaly in patients with myelofibrosis: a case series. Clin Lymphoma Myeloma Leuk. 2018;18 (11):e463-e468. doi:10.1016/j.clml.2018.06.025

55. Palandri F, Tiribelli M, Breccia M, et al. Ruxolitinib rechallenge in resistant or intolerant patients with myelofibrosis: frequency, therapeutic effects, and impact on outcome. Cancer. 2021;127 (15):2657-2665. doi:10.1002/cncr.33541

56. Tremblay D, Mascarenhas J. Next generation therapeutics for the treatment of myelofibrosis. Cells. 2021;10(5):1034. doi:10.3390/ cells 10051034
OncoTargets and Therapy

\section{Publish your work in this journal}

OncoTargets and Therapy is an international, peer-reviewed, open access journal focusing on the pathological basis of all cancers, potential targets for therapy and treatment protocols employed to improve the management of cancer patients. The journal also focuses on the impact of management programs and new therapeutic agents and protocols on patient perspectives such as quality of life, adherence and satisfaction. The manuscript management system is completely online and includes a very quick and fair peer-review system, which is all easy to use. Visit http://www.dovepress.com/ testimonials.php to read real quotes from published authors. 